\title{
Is dermoscopy a good tool for the diagnosis of lentigo maligna and lentigo maligna melanoma? A meta-analysis
}

This article was published in the following Dove Press journal: Clinical, Cosmetic and Investigational Dermatology

\section{Murilo de Oliveira Lima Carapeba ${ }^{1,2}$ Mariana Alves Pineze ${ }^{2}$ Gisele Alborghetti Nai ${ }^{2,3}$ \\ 'Department of Dermatology, Universidade do Oeste Paulista (UNOESTE), Presidente Prudente, SP 19050-680, Brazil; ${ }^{2}$ Medical School, Universidade do Oeste Paulista (UNOESTE), Presidente Prudente, SP, 19050-680, Brazil; ${ }^{3}$ Department of Pathology, Universidade do Oeste Paulista (UNOESTE), Presidente Prudente, SP, 19050-680, Brazil}

\section{Video abstract}

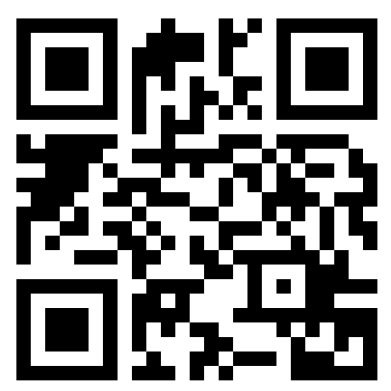

Point your SmartPhone at the code above. If you have a $Q R$ code reader the video abstract will appear. Or use: https://youtu.be/QGHCmdQ8Q04

Correspondence: Gisele Alborghetti Nai Laboratório de Anatomia Patológica e Citopatologia, Universidade do Oeste Paulista (UNOESTE), Rua José

Bongiovani, 700, Presidente Prudente, SP 19050-680, Brazil

Tel +55 I83229 1059

Fax $+55183229 \quad 1194$

Email patologia@unoeste.br

Introduction: Dermoscopy is a low-cost examination performed by a dermatologist and good for the diagnosis of pigmented lesions. However, dermoscopy diagnosis of lentigo maligna (LM) and lentigo maligna melanoma (LMM) is still questionable. The objective of this study was to evaluate whether dermoscopy is an effective diagnostic method to diagnose LM/LMM from other pigmented skin lesions, and to identify which are the most frequent dermoscopic criteria associated with LM/LMM

Methods: For this systematic review and meta-analysis, we used the following descriptors: dermoscopy, lentigo maligna, lentigo maligna melanoma, histopathology; and the following databases to search for articles: Cochrane Collaboration, MEDLINE; PMC (PubMed Central) NIH (National Institutes of Health), EMBASE (The Excerpt Medical Database), and SCISEARCH, from inception to March 30, 2018. The evaluation of studies was performed using the QUADAS (Quality Assessment of Diagnostic Accuracy Studies)-2 tool. The PRISMA (Preferred Reporting Itens for Systematic Reviews and Meta-Analyses) and MOOSE (Meta-analysis Of Observational Studies in Epidemiology) guidelines were followed for data extraction. Also, we extracted from each study the dermoscopic criteria most commonly found in the lesions of LM/LMM.

Results: This systematic review included 15 articles for qualitative analysis (a total of 2,012 lesions evaluated) and 7 for meta-analysis. In the bivariate model the mean sensitivity was 0.824 and the mean specificity was 0.835 . The area under the curve was 0.889 . Rhomboid structures, pseudonetwork, and homogeneous areas were the most frequent dermoscopic criteria associated with LM/LMM.

Conclusion: These findings suggest that dermoscopy has good accuracy in the diagnosis of LM/ LMM.

Keywords: melanoma, diagnosis, systematic review, histopathology, biopsy

\section{Introduction}

Lentigo maligna melanoma (LMM) accounts for $10 \%$ of the malignant melanomas and describes when lentigo maligna (LM) acquires a vertical invasive phase. ${ }^{1}$ LM is considered an in situ melanoma, and in its evolution of atypical melanocytes that invade the dermis, it is characterized as LMM. LM occurs in chronically sun-damaged skin, has slow growth, prevalent in patients aged over 40 years and has a malignant progression rate of approximately $5-20 \%$ of the total. ${ }^{2}$

The gold standard for the diagnosis of LM and LMM should be made using biopsy with distinct histopathological characteristics. These include a pagetoid appearance of 


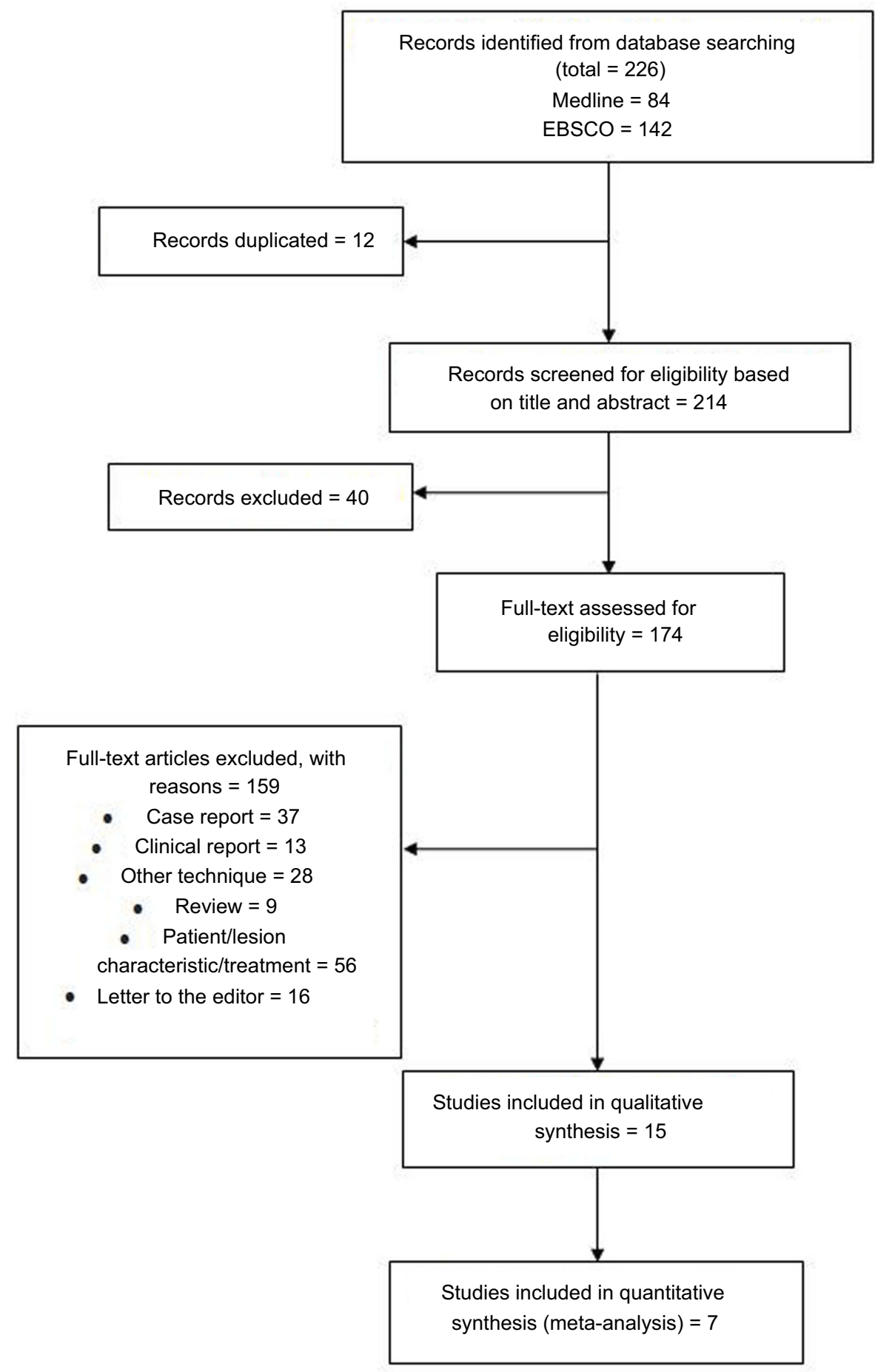

Figure I Search strategy.

melanocytes, melanocyte atypia, nonuniform pigmentation/ distribution of melanocytes, and increased melanocyte density in a background of extensive photodamage. ${ }^{2}$

Dermoscopy is a low-cost examination performed by a dermatologist that is the most useful technique to study melanocytic skin lesions in the differentiation of benign lesions from malignant lesions. It is the most cost-effective examination that can direct the dermatologist to the best site for biopsy. It can also be very useful in the demarcation of lesion limits such as those of LM/LMM and to follow-up and perform cure control of these lesions. ${ }^{1}$

The LM/LMM diagnosis based on dermoscopy is ambiguous. For example, asymmetric pigmented follicular openings, hyperpigmentation of the follicle rim, light 
brown pseudonetworks, light rhomboidal structures, light streaks, and peripheral gray dots could be observed in actinic keratosis and in LM/LMM. ${ }^{2}$

The importance of establishing dermoscopic criteria for the diagnosis of LM/LMM is due to the correct selection of treatment, to minimize the damage of invasive procedures and reduce the associated morbidity and costs of unnecessary surgeries. In some cases, dermoscopy may be a cheap screening test for other noninvasive diagnostic techniques, such as confocal dermoscopy, optical coherence tomography, and high-frequency ultrasonography. ${ }^{3}$

Information from systematic reviews of diagnostic tests is important to determine the appropriate and effective use of diagnostic tests in clinical practice and to develop information necessary to determine the directions of future research in diagnostic medicine. ${ }^{4}$

The aim of this study was to evaluate whether dermoscopy is an effective diagnostic method to differentiate LM and LMM from other pigmented skin lesions and to identify which are the most frequent dermoscopic criteria associated with LM/LMM.

\section{Methods}

The following question was asked to evaluate the role of dermoscopy in the diagnosis of lentigo maligna and lentigo maligna melanoma on dermatological examination: What is the efficacy of dermoscopy in the diagnosis of lentigo maligna and lentigo maligna melanoma compared to histopathology?

\section{Data source and search strategy}

To address the issue, we conducted detailed and automated research using the following databases to search for articles, without any restrictions regarding the date, language or any other parameter: Cochrane Collaboration; MEDLINE; PMC (PubMed Central) - NIH (National Institutes of Health); EMBASE (The Excerpt Medical Database); SCISEARCH from inception of these databases to March 30, 2018.

The search strategy of the articles was as follows: "Lentigo" OR "Hutchinson's Melanotic Freckle" OR Lentigos OR Lentigines OR Lentiginosis OR Lentiginoses OR (Freckle, Hutchinson's Melanotic) OR (Hutchinson Melanotic Freckle) OR (Hutchinsons Melanotic Freckle) OR (Melanotic Freckle, Hutchinson's) OR (Melanotic Freckle) OR (Lentigo, Malignant) OR (Lentigos, Malignant) OR (Malignant Lentigo) OR (Malignant Lentigos) OR (Freckle, Melanotic) OR (Freckles, Melanotic) OR (Melanotic
Freckles) OR (Lentigo Maligna) AND "Dermoscopy" OR Dermoscopies OR Dermatoscopy OR Dermatoscopies OR (Skin Surface Microscopy) OR (Microscopies, Skin Surface) OR (Microscopy, Skin Surface) OR (Skin Surface Microscopies) OR (Surface Microscopies, Skin) OR (Surface Microscopy, Skin) OR (Epiluminescence Microscopy) OR (Epiluminescence Microscopies) OR (Microscopies, Epiluminescence) OR (Microscopy, Epiluminescence) AND "Biopsy" OR Biopsies AND 'Histopathology'.

\section{Eligibility criteria and study selection}

The studies with a grade of recommendation A or B were selected according to the document "Levels of Evidence 1" of the CEBM (Center for Evidence-based Medicine), ${ }^{5}$ and the $\mathrm{C}$ or D studies listed in this document were excluded.

\section{Quality assessment of included studies}

The evaluation of the studies was performed using the QUADAS (Quality Assessment of Diagnostic Accuracy Studies)-2 tool, recommended for systematic reviews of diagnostic accuracy by the Agency for Healthcare Research and Quality, Cochrane Collaboration, and UK National Institute for Health and Clinical Excellence. ${ }^{6}$

\section{Data extraction}

Two authors (MOLC and MAP) independently assessed the titles and abstracts of all studies identified in the electronic search. In the event of disagreement, a consensus was reached. From this action, a collection of studies that affirmatively answered our question was created that was evaluated in their entirety by the authors.

From each study were extracted the tests used to evaluate the lesions, which lesions were evaluated and their location and the number of cases evaluated. The general sensitivity and specificity of dermoscopy were extracted for meta-analysis.

Additionally, we extracted from each study the dermoscopic criteria most commonly found in the lesions of LM/LMM.

\section{Definitions}

- The definitions for sensitivity, specificity, likelihood ratio (LR), likelihood ratio for a positive test, likelihood ratio for a negative test, and diagnostic odds ratio (DOR) can be found in the article by Šimundić. ${ }^{7}$ 


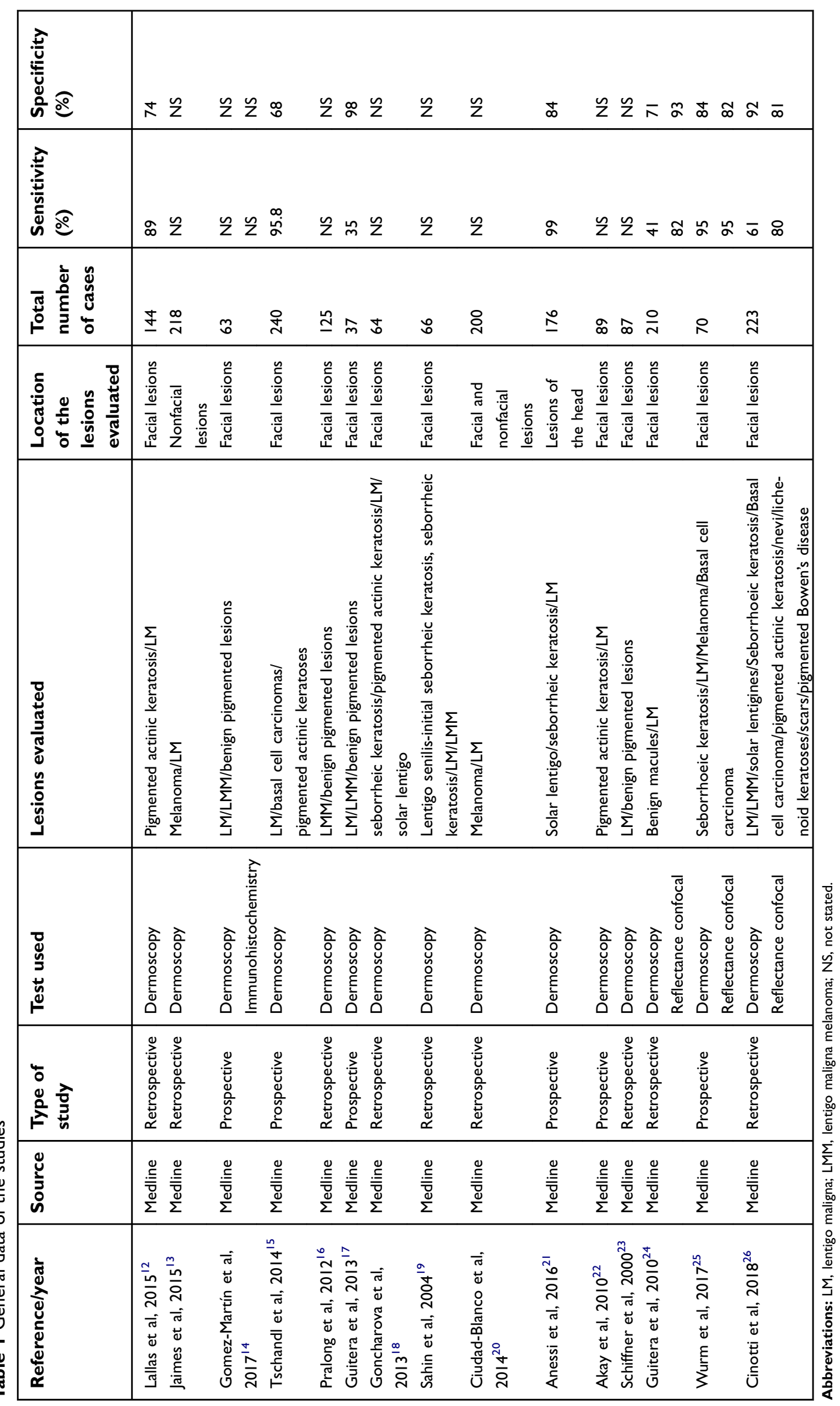




\section{Meta-analysis}

The heterogeneity among the included studies was assessed using the $\mathrm{Q}$ test for statistical significance and the I-square test to quantify heterogeneity, where $p<0.1$ is statistically significant and I-square $>25 \%$ shows significant heterogeneity. The corresponding 95\% confidence intervals (CI) were also estimated.

The receiver operating characteristic (ROC) curve was used to summarize the results of the studies. The area under the curve (AUC), which summarizes the diagnostic performance, was calculated. A perfect test has an AUC close to 1, and poor tests have AUCs close to 0.5 . The index $\mathrm{Q}^{*}$, defined by the point where the sensitivity and specificity are equal, is the nearest point of the ideal in the upper left corner of the ROC curve (specificity $=0$, sensitivity $=1$ ), and the standard errors of AUC [SE (AUC)] and Q * [SE (Q *)] were also calculated. The statistical tests were performed using Meta-Disc software. ${ }^{8}$

The bivariate model of Reitsma et $\mathrm{al}^{9}$ was used to enhance the understanding of the heterogeneity of results between studies and the correlation within studies. For the bivariate analysis, we used the Software R v. 3.5.3 with the package MADA (Meta-Analysis of Diagnostic Accuracy) v. 0.5.8.

All statistical tests were performed with a significance level of $5 \%$.

\section{Results}

In the databases searched for the period from 1996 to 2018, 226 articles were found, from which 15 were included for qualitative analysis among a total of 2,012 lesions evaluated.

Figure 1 shows the search strategy of the articles based on the PRISMA (Preferred Reporting Itens for Systematic Reviews and Meta-Analyses) ${ }^{10}$ and MOOSE (Meta-analysis Of Observational Studies in Epidemiology) ${ }^{11}$ guidelines. Table 1 summarizes the characteristics of each study.

Regarding the quality evaluation of the 15 studies, only 2 studies ${ }^{15,23}$ presented an uncertain/high risk of bias in relation to the index test (dermoscopy); most of the included studies had a low risk of bias with respect to the index test and patient choice and flow and time (Table 2).

Of the 15 included studies comparing dermoscopy and histopathology, from 7 studies, ${ }^{12,15,17,21,24-26}$ it was possible to extract data on the general specificity and sensitivity of dermoscopy. These seven studies were included in the quantitative analysis (meta-analysis).
The random-effects model was used for the combination of specificity and sensitivity. Cochran's Q statistic for heterogeneity showed the heterogeneity between the studies, both for specificity $(p<0.001)$ and sensitivity $(p<0.001)$, and that heterogeneity is large for specificity [I-square $\left(\mathrm{I}^{2}\right)$ $=91.2 \%]$ and for sensitivity $\left(\mathrm{I}^{2}=96.3 \%\right)$. The pooled sensitivity was 0.71 (95\% CI: $0.67-0.76)$, and the pooled specificity was $0.81 \quad(95 \%$ CI: $0.78-0.84)$ (Figure 2). The I-square value for the positive likelihood ratio (LR) was $84.1 \%$, that for the negative LR was $94.4 \%$ and that for the diagnostic odds ratio (DOR) was $86.9 \%$. The pooled positive LR was 4.352 (95\% CI: 2.734-6.925), the pooled negative LR was 0.248 (95\% CI: $0.122-0.503)$, and the pooled DOR was 28.47 (95\% CI: 7.859-102.68). The accuracy of the test, as measured by the area under the curve (AUC), was 0.9147 (SE=0.0358) (Figure 3).

In the bivariate model, mean sensitivity was 0.824 (95\% CI: $0.531-0.951)$, mean specificity was 0.835 (95\% CI: $0.720-0.909)$, and mean DOR was 30.400 (95\% CI: 6.880-92.400). The AUC was 0.889 and the partial AUC (restricted to observed false-positive results and normalized) was 0.712 (Figure 4).

\section{Dermoscopic criteria}

It was possible to extract the incidence of the dermoscopic criteria used for the diagnosis of LM/LMM from eleven included studies. The most frequent criteria in each study are described in Table 3.

The common dermoscopic criteria most observed in all studies were rhomboidal structures, described in four studies $^{12,14,19,22}$ (56-75\% of the cases presented the criteria); pseudonetwork, described in three studies ${ }^{14,15,18}$ (71-87.5\% of the cases presented the criteria); homogeneous areas, described in three studies ${ }^{18,19,23}(54.5-75 \%$ of the cases presented the criteria); black points, in two articles ${ }^{19,22}$ (86.4-100\% of the cases); blue-gray dots and globules, in two articles ${ }^{19,22}$ (60.8-95.8\% of the cases); brown color in two articles ${ }^{12,19}$ (72.7-90\% of the cases); and gray circles, in two articles ${ }^{12,19}(54.2-56 \%$ of the cases).

Two studies ${ }^{12,15}$ analyzed the specificity, sensitivity, and relative risk of the main dermoscopic criteria found. In the study by Tschandl et al, ${ }^{15}$ the most sensitive dermoscopic criteria were any gray structure $(95.8 \%$ CI: $78.8-99.3 \%)$, with a specificity of $30.6 \%$ (CI: $24.5-37.2 \%$ ), dot vessels, with a higher specificity (98.1\% CI: 95.3-99.5\%) and a sensitivity of $8.3 \%$ (CI: $1.3-27.0 \%$ ), and circle in a circle/ double circle, with a specificity of $98.1 \%$ (CI: $95.3-99.5 \%$ ) and sensitivity of $4.2 \%$ (CI: $0.7-21.2 \%$ ). In the study of Lallas 


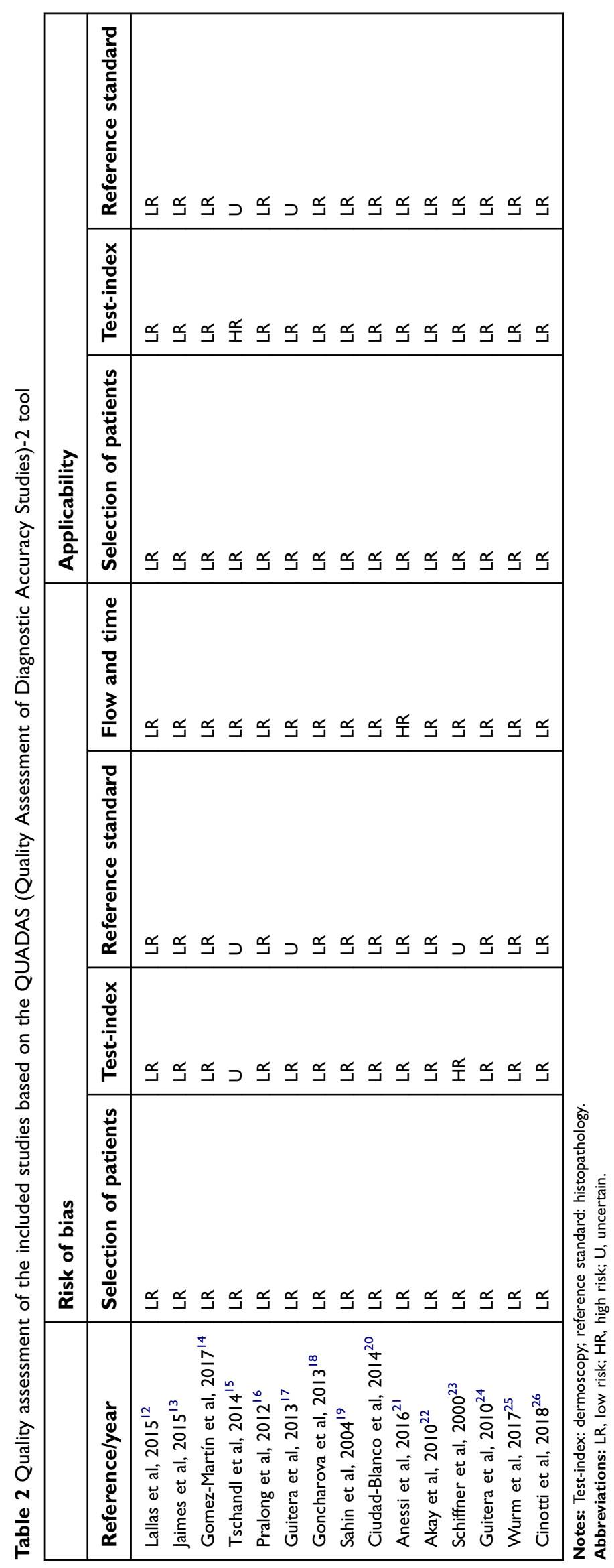




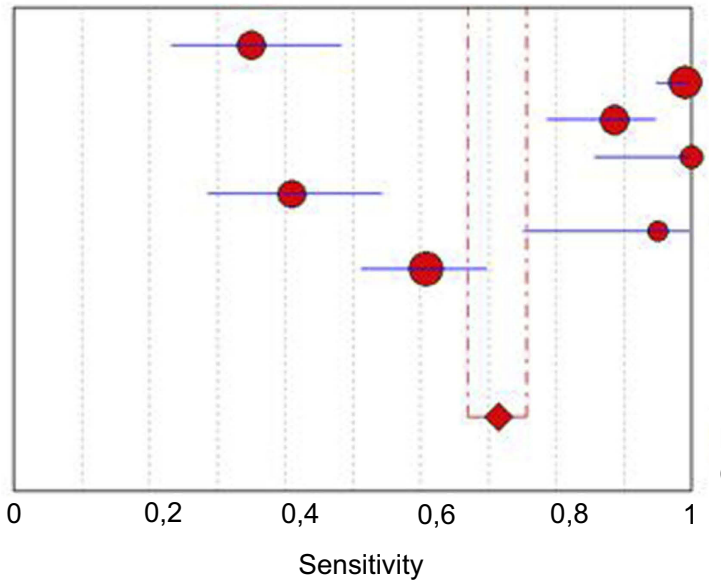

Guitera2013

Annessi2016

Lallas2015

TschandI2014

Guitera2010

Wurm2017

Cinotti2018

Pooled sensitivity $=0,71(0,67$ to 0,76$)$

Chi-square $=161,22 ; \mathrm{df}=6(P=0,0000)$

Inconsistency ( $($-square) $=96,3 \%$

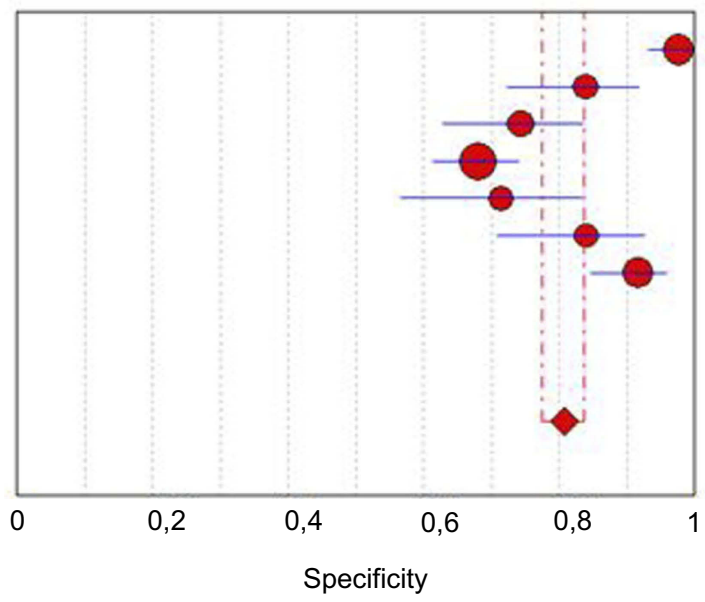

Sensitivity $(95 \% \mathrm{Cl})$

$0,35 \quad(0,23-0,48)$

$0,99 \quad(0,99-1,00)$

$0,89 \quad(0,79-0,95)$

$1,00 \quad(0,86-1,00)$

$0,41 \quad(0,29-0,54)$

$0,95 \quad(0,75-1,00)$

$0,61 \quad(0,51-0,70)$
Guitera2013

Annessi2016

Lallas2015

Tschandl2014

Guitera2010

Wurm2017

Cinotti2018
Specificity $(95 \% \mathrm{Cl})$

$0,98 \quad(0,93-1,00)$

$0,84 \quad(0,72-0,92)$

$0,74 \quad(0,63-0,84)$

$0,68 \quad(0,61-0,74)$

$0,71 \quad(0,57-0,83)$

$0,84 \quad(0,71-0,93)$

$0,92 \quad(0,85-0,96)$

Pooled specificity $=0,81(0,78$ to 0,84$)$

Chi-square $=68,45 ; \mathrm{df}=6(P=0,0000)$

Inconsistency ( $I$-square) $=91,2 \%$

Figure 2 Diagram of the meta-analysis for the sensitivity and specificity of dermoscopy. Abbreviations: $95 \% \mathrm{Cl}, 95 \%$ confidence interval; df, degrees of freedom.

et $\mathrm{al},{ }^{12}$ the dermoscopic criteria that presented the highest relative risk were not evident follicles (6.33; CI: 3.06-12.98) and gray circles (5.9; CI: 2.76-12.65).

\section{Discussion}

In this meta-analysis, dermoscopy showed a good accuracy in the diagnosis of LM/LMM, demonstrated by AUC between 0.8 and 0.9 in the bivariate model. Also rhomboid structures, followed by pseudonetwork and homogeneous areas were the most observed dermoscopic criteria in LM/ LMM in the studies analyzed.

The effectiveness of the use of dermoscopy depends on the professional conducting the procedure because the criteria used for the diagnosis vary among several authors, as well as the interpretation of these criteria during the examination. Therefore, studies that described the dermoscopic analysis without revision by another dermatologist were considered to harbor a risk of uncertain/high bias in relation to the index test (dermoscopy). Only two studies $^{15,23}$ presented an uncertain/high risk of bias in relation to the index test (dermoscopy).

Among the included studies, we observed that only one differentiated between the LMM and LMM dermoscopic criteria, ${ }^{23}$ suggesting a tendency to study such diseases as the same entity or perhaps because of the difficulty of dermoscopic differentiation of both using the existing criteria.

Among the seven studies ${ }^{12,15,17,21,24-26}$ that described the general sensitivity and specificity, dermoscopy for LM/ LMM showed a pooled sensitivity of 0.71 and pooled specificity of 0.81 . The pooled positive LR was 4.352 , the pooled negative LR was 0.248 , and the pooled DOR was 28.407. Good diagnostic tests have positive LRs greater than 10 and negative LRs less than $0.1 .^{7}$ However, diagnostic tests with AUC between 0.9 and 1.0 


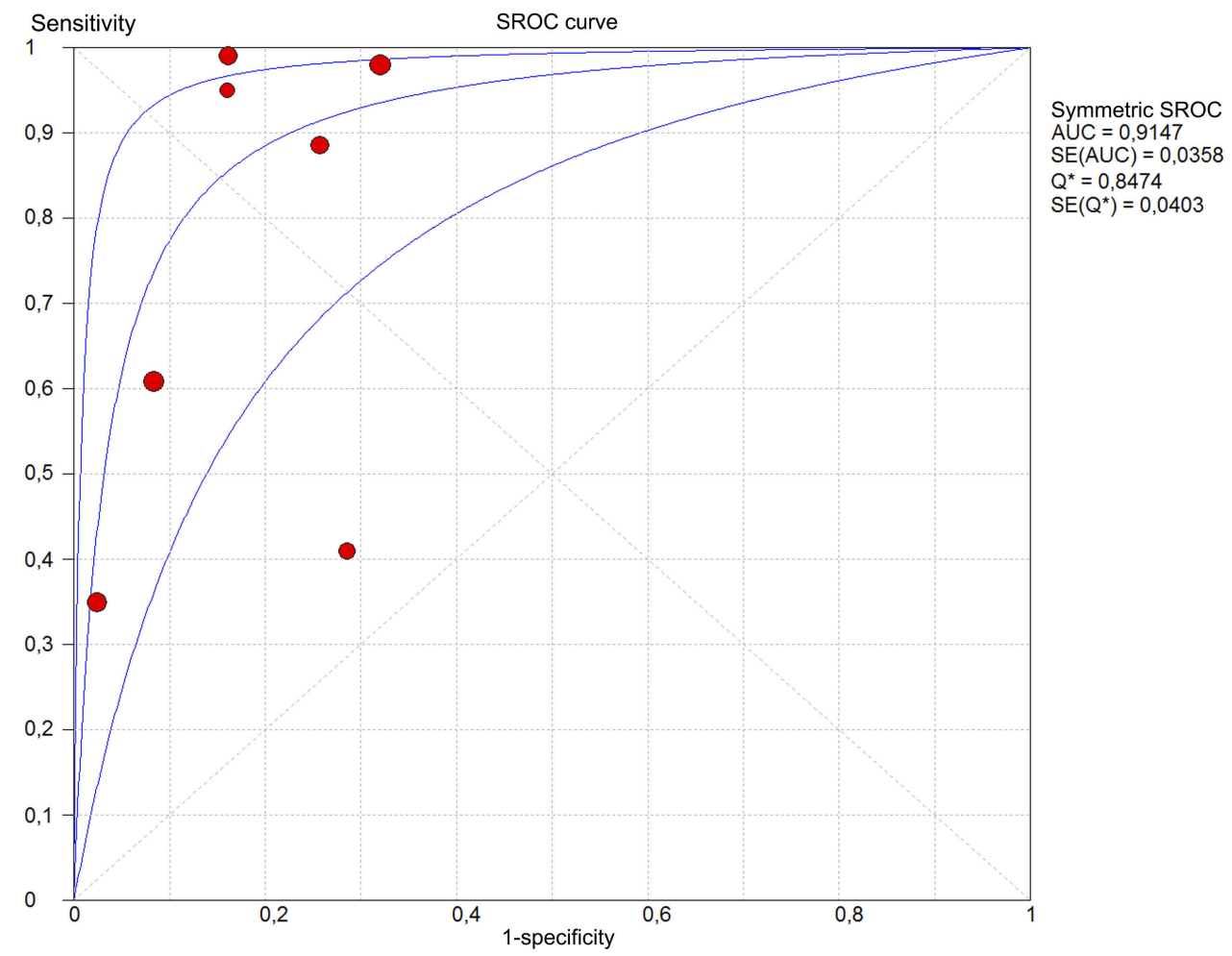

Figure 3 Estimated SROC (Summary Receiver Operating Characteristics) curve and original data points for dermoscopy compared with histopathology. Abbreviations: AUC, area under the curve; SE (AUC), standard errors of $\mathrm{AUC}$; $\mathrm{Q}^{*}$, chi-squared; $\mathrm{SE}\left(\mathrm{Q}^{*}\right)$, standard errors of $\mathrm{Q}^{*}$.

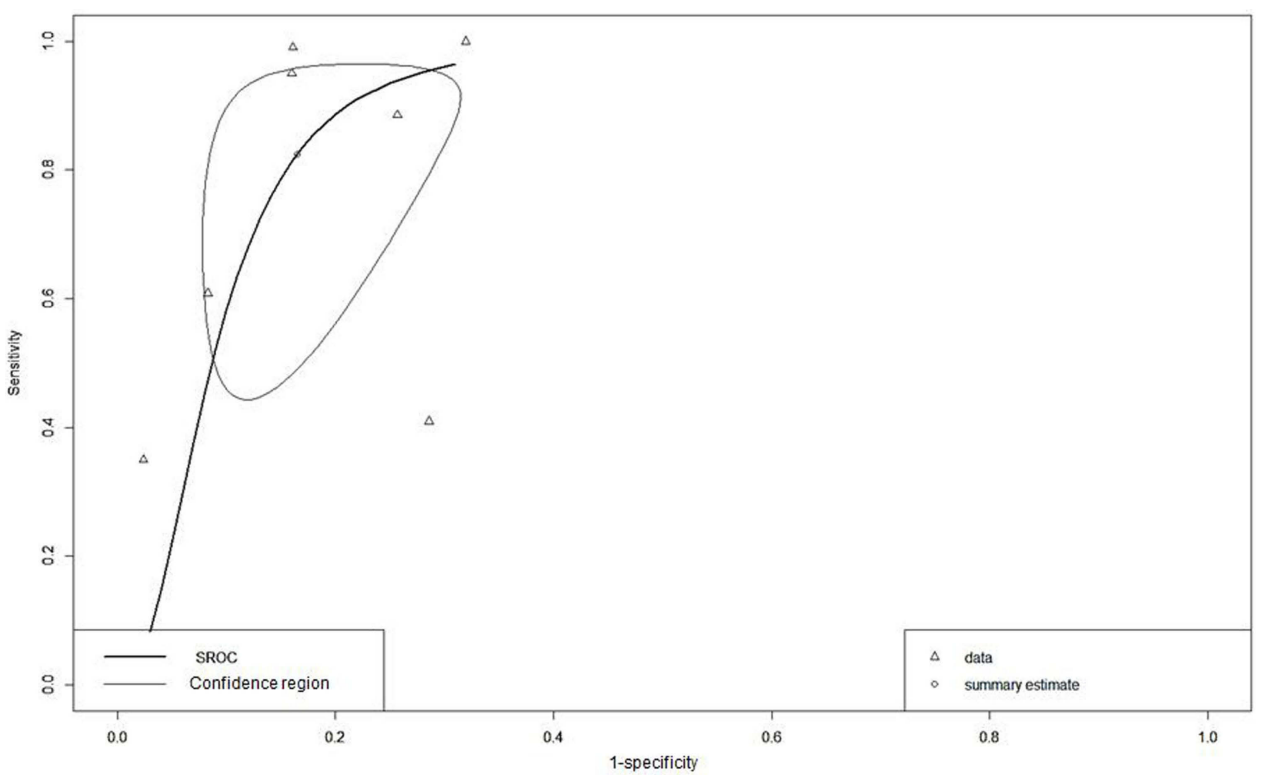

Figure 4 Estimated SROC (Summary Receiver Operating Characteristics) curve (Bivariate model) and the corresponding 95\% confidence ellipse.

have excellent diagnostic accuracy; ${ }^{7}$ in our study, the AUC was 0.9147. Although the pooled positive LR and the pooled DOR did not show favorable indices for dermatoscopy, the pooled sensitivity, the pooled specificity, the pooled negative LR, and AUC favor that dermoscopy is a good test for the diagnosis of LM/LMM.

But the studies included in this meta-analysis present some general limitations in its design, such as the hetero- 
Table 3 Dermoscopic criteria occurring in 50\% or more of the LM/LMM lesions in eleven selected studies

\begin{tabular}{|c|c|c|c|}
\hline Reference/year & Dermoscopic criteria & \multicolumn{2}{|c|}{$\begin{array}{l}\text { Number of LM/LMM cases that presented the } \\
\text { criteria (\%) }\end{array}$} \\
\hline \multirow[t]{4}{*}{ Lallas et al, $2015^{12}$} & Gray color & \multicolumn{2}{|l|}{$63(90)$} \\
\hline & Brown color & \multicolumn{2}{|l|}{$63(90)$} \\
\hline & Gray circles & \multicolumn{2}{|l|}{$39(56)$} \\
\hline & Gray rhombic lines & \multicolumn{2}{|l|}{$39(56)$} \\
\hline \multirow[t]{10}{*}{ Jaimes et al, $2015^{13}$} & & LM & LMM \\
\hline & Granulation or peppering & $98(69)$ & $25(64.1)$ \\
\hline & Light brown color & $137(96.5)$ & $38(97.4)$ \\
\hline & Dark brown color & $130(9 \mid .5)$ & $36(92.3)$ \\
\hline & Bluish gray & $94(66.2)$ & $31(79.5)$ \\
\hline & Blue whitish & $42(29.6)$ & $2 \mathrm{I}(53.8)$ \\
\hline & Vases on stitch & - & $22(56.4)$ \\
\hline & Vascular blush & - & $2 I(53.8)$ \\
\hline & Pink color & - & $26(66.7)$ \\
\hline & White color & - & $22(56.4)$ \\
\hline \multirow[t]{3}{*}{ Gomez-Martim et al, $2017^{14}$} & Asymmetric pigmented follicular openings & \multicolumn{2}{|l|}{$16(67)$} \\
\hline & Pseudonetwork & \multicolumn{2}{|l|}{$18(75)$} \\
\hline & Pigmented rhomboidal structures & \multicolumn{2}{|l|}{$18(75)$} \\
\hline Tschandl et al, $2014^{15}$ & Gray structures & \multicolumn{2}{|l|}{$23(95.8)$} \\
\hline \multirow[t]{4}{*}{ Pralong et al, $2012^{16}$} & $3-4$ colors & \multicolumn{2}{|l|}{$78(62)$} \\
\hline & Hyperpigmented follicular opening & \multicolumn{2}{|l|}{$64(5 \mathrm{I})$} \\
\hline & Pigmented follicular opening & \multicolumn{2}{|l|}{$86(69)$} \\
\hline & Increased vascular network density & \multicolumn{2}{|l|}{$72(58)$} \\
\hline \multirow[t]{3}{*}{ Goncharova et al, $2013^{18}$} & Points and globules & \multicolumn{2}{|l|}{$4(50)$} \\
\hline & Pseudonetwork & \multicolumn{2}{|l|}{$7(87.5)$} \\
\hline & Homogeneous structures & \multicolumn{2}{|l|}{$6(75)$} \\
\hline \multirow[t]{8}{*}{ Sahin et al, $2014^{19}$} & Asymmetrical and pigmented follicular openings & \multicolumn{2}{|l|}{$18(81.8)$} \\
\hline & Dark lines & \multicolumn{2}{|l|}{$16(72.7)$} \\
\hline & Dark globules & \multicolumn{2}{|l|}{$19(86.3)$} \\
\hline & Gray globules & \multicolumn{2}{|l|}{$12(54.5)$} \\
\hline & Dark dots & \multicolumn{2}{|l|}{$16(72.7)$} \\
\hline & Dark rhomboidal structures (black and brown) & \multicolumn{2}{|l|}{ II (50) } \\
\hline & Clear rhomboidal structures & \multicolumn{2}{|l|}{$14(63.6)$} \\
\hline & Dark pseudonetwork & \multicolumn{2}{|l|}{$18(81.8)$} \\
\hline
\end{tabular}


Table 3 (Continued).

\begin{tabular}{|c|c|c|}
\hline Reference/year & Dermoscopic criteria & $\begin{array}{l}\text { Number of LM/LMM cases that presented the } \\
\text { criteria (\%) }\end{array}$ \\
\hline Ciudad-Blanco et al, $2014^{20}$ & Dots and blue-gray globules & $31(60.8)$ \\
\hline \multirow[t]{5}{*}{ Anessi et al, $2016^{21}$} & Light brown/dark pseudonetwork & $105(100)$ \\
\hline & Fine pigmented brown net & $76(72.4)$ \\
\hline & Atypical pigmented network & $70(66.7)$ \\
\hline & Blue-gray dots & $88(83.8)$ \\
\hline & Dark brown/blue-gray ribbon structures & $80(76.2)$ \\
\hline \multirow[t]{2}{*}{ Akay et al, $2010^{22}$} & Rhomboidal structures & $15(75)$ \\
\hline & Black blots & $15(75)$ \\
\hline \multirow[t]{7}{*}{ Schiffner et al, $2000^{23}$} & Pigmented asymmetric follicular opening & $25(67.6)$ \\
\hline & Dark lines & $19(5 \mid .3)$ \\
\hline & Dark dots & $32(86.5)$ \\
\hline & Gray dots & $28(75.7)$ \\
\hline & Dark globules & $35(94.6)$ \\
\hline & Gray globules & $31(83.8)$ \\
\hline & Homogeneous dark areas & $23(62.2)$ \\
\hline
\end{tabular}

Abbreviations: LM, lentigo maligna; LMM, lentigo maligna melanoma.

geneity of lesions evaluated, indistinct evaluation between LM and LMM, a small number of cases evaluated in some studies, retrospective studies, and great heterogeneity of the specificity and sensitivity among the studies included in the meta-analysis. Because of this, we performed a bivariate analysis.

In the bivariate model, that estimates the amount of between-study variability in both sensitivity and specificity and evaluates more adequately studies with a lot of heterogeneity, ${ }^{9}$ the mean sensitivity of 0.824 , a mean specificity of 0.835 , and mean DOR of 30.400 . Also, the AUC was 0.889 and the partial AUC was 0.712 . Diagnostic tests with AUC between 0.8 and 0.9 have very good diagnostic accuracy and tests with AUC between 0.7 and 0.8 have good diagnostic accuracy. ${ }^{7}$ These data show that dermoscopy has a good accuracy for the diagnosis of LM/LMM.

Currently, there are classic criteria used in dermoscopy, but several authors have tried to elucidate the diagnoses of these lesions by improving pre-existing standardized criteria $^{25}$ and sometimes introducing new criteria, such as those by Pralong et al, ${ }^{16}$ who introduced four new criteria (darkening in dermoscopic examination, target-like pattern, red rhomboidal structures, and increased vascular network density) and Tschandl et al ${ }^{15}$ (four-dot clods, double circle, incomplete circles, and edge in bite).

Some authors have described that if asymmetric pigmented follicular openings, dark rhomboidal structures, slate-gray areas, and slate-gray dots/globules/pepper pattern are found in one lesion, they have high sensitivity and specificity for a diagnosis of LMM. ${ }^{22,27}$

In the assessment of the dermoscopic criteria, which were identified in more than $50 \%$ of the biopsied LM/ LMM lesions, rhomboidal structures were the dermoscopic criteria that appeared in more studies (four), with an incidence ranging from $56 \%$ to $75 \%$ of the cases, followed by a pseudonetwork (incidence between $71 \%$ and $87.5 \%$ of the cases), and homogeneous areas (incidence between $54.5 \%$ and $75 \%$ of the cases), identified in three articles. Thus, we suggest that the identification of these criteria for dermoscopy favors the diagnosis of LM/LMM. 
Although any gray structure was the most sensitive dermoscopic criteria and dot vessels and circle in a circle/double circle were more specific, the specificity and sensitivity of the dermoscopic criteria were only evaluated in two articles, ${ }^{12,15}$ with a few cases studied that only evaluated LM, preventing adequate extrapolation of these findings to all lentiginous lesions.

This systematic review offers evidence of previously used criteria to diagnose LM and LMM, as described by Stolz et $\mathrm{al}^{27}$ (hyperpigmented follicular opening, annular-granular pattern, pigmented rhomboid structures, and obliterated hair follicles) as well as new criteria used by the authors that can be considered in the evaluation of these lesions by the prevalence of appearance in the studies, such as homogeneous areas, black spots, dots and bluish-gray globules, brown color, and gray circle.

We have to consider that only seven studies ${ }^{12,15,17,21,24-26}$ presented sensitivity and specificity assessment for dermoscopy in the diagnosis of LM/LMM, and only two ${ }^{12,15}$ evaluated the sensitivity and specificity of dermoscopic criteria. So, further studies evaluating the sensitivity and specificity of the dermoscopic criteria for LM/LMM may contribute to the improvement of the dermoscopic diagnosis of these lesions.

\section{Conclusion}

On the basis of this study, dermoscopy has good accuracy in the diagnosis of LM/LMM compared to histopathology and can be used for define the optimal biopsy site or even for setting the total removal of the lesion. Also the identification of rhomboidal structures, pseudonetwork, and homogeneous areas are the criteria that seem most favor the diagnosis of LM/LMM.

\section{Acknowledgment}

The authors would like to thank Mrs Jakeline MQ Ortega, from the Library Division of the Universidade do Oeste Paulista, for her assistance during the initial selection of articles.

\section{Disclosure}

The authors report no conflicts of interest in this work.

\section{References}

1. Edwards SJ, Osei-Assibey G, Patalay R, Wakefield V, Karner C. Diagnostic accuracy of reflectance confocal microscopy using VivaScope for detecting and monitoring skin lesions: a systematic review. Clin Exp Dermatol. 2017;42:266-275. doi:10.1111/ced.13055
2. Juhász MLW, Marmur ES. Reviewing challenges in the diagnosis and treatment of lentigo maligna and lentigo-maligna melanoma. Rare Cancers Ther. 2015;3:133-145. doi:10.1007/s40487-0150012-9

3. Kasprzak JM, Xu YG. Diagnosis and management of lentigo maligna: a review. Drugs Context. 2015;4:212281. doi:10.7573/dic.212281

4. Gatsonis C, Paliwal P. Meta-analysis of diagnostic and screening test accuracy evaluations: methodologic primer. AJR. 2006;187:271-281. doi:10.2214/AJR.06.0226

5. OCEBM Levels of Evidence Working Group. The Oxford Levels of Evidence 2. Oxford Centre for Evidence-Based Medicine; 2016. Available from: https://www.cebm.net/index.aspx?o=5653. Accessed April 17, 2017.

6. Whiting PF, Rutjes AW, Westwood ME, et al; QUADAS-2 Group. QUADAS-2: a revised tool for the quality assessment of diagnostic accuracy studies. Ann Intern Med. 2011;155:529-536. doi:10.7326/ 0003-4819-155-8-201110180-00009

7. Šimundić AM. Measures of diagnostic accuracy: basic definitions. EJIFCC. 2009;19:203-211.

8. Zamora J, Abraira V, Muriel A, Khan KS, Coomarasamy A. MetaDiSc: a software for meta-analysis of test accuracy data. BMC Med Res Methodol. 2006;6:31. doi:10.1186/1471-2288-6-31

9. Reitsma JB, Glas AS, Rutjes AW, Scholten RJ, Bossuyt PM, Zwinderman AH. Bivariate analysis of sensitivity and specificity produces informative summary measures in diagnostic reviews. J Clin Epidemiol. 2005; 58(10):982-990. doi:10.1016/j.jclinepi.2005.02.022

10. Moher D, Liberati A, Tetzlaff J, Altman DG, The PRISMA Group. Preferred reporting items for systematic reviews and meta-analyses: The PRISMA Statement. PLoS Med. 2009;6:e1000097. doi:10.1371/ journal.pmed1000097

11. Stroup DF, Berlin JA, Morton SC, Olkin I, Williamson GD, Rennie D, et al. Meta-analysis of observational studies in epidemiology: a proposal for reporting. Meta-analysis of observational studies in epidemiology (MOOSE) group. JAMA. 2000;283:2008-2012. doi:10.1001/jama.283.15.2008

12. Lallas A, Tschandl P, Kyrgidis A, et al. Dermoscopic clues to differentiate facial lentigo maligna from pigmented actinic keratosis. $\mathrm{Br}$ J Dermatol. 2016;174:1079-1085. doi:10.1111/bjd.14355

13. Jaimes N, Marghoob AA, Rabinovitz H, et al. Clinical and dermoscopic characteristics of melanomas on nonfacial chronically sun-damagedskin. J Am Acad Dermatol. 2015;72:1027-1035. doi:10.1016/j.jaad.2015.02.1117

14. Gomez-Martin I, Moreno S, Andrades-Lopez E, et al. Histopathologic and immunohistochemical correlates of confocal descriptors in pigmented facial macules on photodamaged skin. JAMA Dermatol. 2017;153:771-780. doi:10.1001/jamadermatol.2017.1323

15. Tschandl P, Rosendahl C, Kittler H. Dermatoscopy of flat pigmented facial lesions. J Eur Acad Dermatol Venereol. 2015;29:120-127. doi:10.1111/jdv.12483

16. Pralong P, Bathelier E, Dalle S, Poulalhon N, Debarbieux S, Thomas L. Dermoscopy of lentigo maligna melanoma: report of 125 cases. Br J Dermatol. 2012;167:280-287. doi:10.1111/j.13652133.2012.10932.x

17. Guitera P, Moloney FJ, Menzies SW, et al. Improving management and patient care in lentigo maligna by mapping with in vivo confocal microscopy. JAMA Dermatol. 2013;149:692-698. doi:10.1001/ jamadermatol.2013.2301

18. Goncharova Y, Attia EAS, Souid K, Vasilenko IV. Dermoscopic features of facial pigmented skin lesions. ISRN Dermatol. 2013;2013:546813. doi:10.1155/2013/546813

19. Sahin MT, Öztürkcan S, Ermertcan AT, Günes AT. A comparison of dermoscopic features among lentigo senilis/initial seborrheic keratosis, seborrheic keratosis, lentigo maligna and lentigo maligna melanoma on the face. $J$ Dermatol. 2004;31:884-889. 
20. Ciudad-Blanco C, Avilés-Izquierdo JA, Lázaro-Ochaita P, SuárezFernández R. Dermoscopic findings for the early detection of melanoma: an analysis of 200 cases. Actas Dermosifiliogr. 2014;105:683-693. doi:10.1016/j.ad.2014.01.008

21. Annessi G, Bono R, Abeni D. Correlation between digital epiluminescence microscopy parameters and histopathological changes in lentigo maligna and solar lentigo: A dermoscopic index for the diagnosis of lentigo maligna. $J$ Am Acad Dermatol. 2017;76:234-243. doi:10.1016/j.jaad.2016.08.032

22. Akay BN, Kocyigit P, Heper AO, Erdem C. Dermatoscopy of flat pigmented facial lesions: diagnostic challenge between pigmented actinic keratosis and lentigo maligna. Br J Dermatol. 2010;163:1212-1217. doi:10.1111/j.1365-2133.2010.10025.x

23. Schiffner R, Schiffner-Rohe J, Vogt T, et al. Improvement of early recognition of lentigo maligna using dermatoscopy. J Am Acad Dermatol. 2000;42:25-32.
24. Guitera P, Pellacani G, Crotty KA, et al. The impact of in vivo reflectance confocal microscopy onthe diagnostic accuracy of lentigo maligna and equivocal pigmented and nonpigmented macules of the face. $J$ Invest Dermatol. 2010;130:2080-2091. doi:10.1038/jid.2010.84

25. Wurm E, Pellacani G, Longo C, et al. The value of reflectance confocal microscopy in diagnosis of flat pigmented facial lesions: a prospective study. $J$ Eur Acad Dermatol Venereol. 2017;31:1349-1354. doi:10.1111/jdv.14171

26. Cinotti E, Labeille B, Debarbieux S, et al. Dermoscopy vs. reflectance confocal microscopy for the diagnosis of lentigo maligna. J Eur Acad Dermatol Venereol. 2018;32:1284-1291. doi:10.1111/ jdv. 14791

27. Stolz W, Schaffner R, Burgdorf WH. Dermatoscopy for facial pigmented skin lesions. Clin Dermatol. 2002;20:276-278.

\section{Publish your work in this journal}

Clinical, Cosmetic and Investigational Dermatology is an international, peer-reviewed, open access, online journal that focuses on the latest clinical and experimental research in all aspects of skin disease and cosmetic interventions. This journal is indexed on CAS.
The manuscript management system is completely online and includes a very quick and fair peer-review system, which is all easy to use. Visit http://www.dovepress.com/testimonials.php to read real quotes from published authors. 